\title{
Current State and Perspective of Turbulent Combustion Research*
}

\author{
Toshio MIYAUCHI** and Mamoru TANAHASHI ${ }^{* *}$ \\ ** Department of Mechanical and Aerospace Engineering, Tokyo Institute of Technology \\ 2-12-1 Ookayama, Meguro-ku, Tokyo 152-8550, Japan \\ E-mail: tmiyauch@mes.titech.ac.jp
}

\begin{abstract}
Recent progresses in turbulent combustion researches are summarized in this paper with a focus on direct numerical simulation (DNS), large eddy simulation (LES) and laser diagnostics of turbulent combustion. In DNS studies of turbulent combustion, detailed kinetic mechanism tends to be included to investigate turbulence/flame interactions. LES of turbulent combustion can be classified into flame tracking method, PDF method and flame thickened method, and will play important roles in the developments of many combustors in engineering applications in near future. In the laser diagnostics of turbulent combustion, simultaneous measurements of several radical concentrations by planer laser induced fluorescence (PLIF) and multi-components velocity measurement by particle image velocimetry (PIV) are effective to investigate the local flame structure in turbulence. The simultaneous time-resolved PLIF and PIV will have great importance in future researches on turbulent combustion.
\end{abstract}

Key words : Turbulent Combustion, Direct Numerical Simulation, Large Eddy Simulation, Planer Laser Induced Fluorescence, Particle Image Velocimetry

\section{Introduction}

Due to recent environmental problems, high efficiency and low emission combustion technology has been required for realistic combustors such as gas turbine and internal combustion engines. Since flows in lots of combustors in engineering applications are in turbulent state, interaction between turbulence and flame induces quite complicated phenomena. However, details of turbulent combustion have not been clarified. Therefore, lots of studies on turbulent combustion have been conducted based on theoretical, experimental and numerical approaches. With the recent developments in the computer technology, numerical simulations have been applied for design and development of many combustors in engineering applications extensively. However, accuracy of the numerical simulations strongly depends on turbulent combustion models and is not always enough. To improve the accuracy of numerical expectations, developments of appropriate turbulent combustion models are required by investigating details of turbulent combustion. In this paper, current state and perspective of direct numerical simulation (DNS), large eddy simulation (LES) and laser diagnostics of turbulent combustion is reviewed.

\section{Turbulent Combustion Diagram}

Characteristics of turbulent flame have been classified based on the characteristic velocity and length scales of turbulent flame similar to the method of dimensional analysis for turbulence. In this paper, fundamental ideas of this classification are presented for turbulent premixed flames. In general, it has been considered that structure of turbulent premixed flame can be classified based on the ratio of turbulence intensity $\left(u_{r m s}^{\prime}\right)$ to laminar burning velocity $\left(S_{L}\right)$ and that of integral length scale of turbulence to laminar flame thickness $\left(\delta_{F}\right)$. Figure 1 shows the turbulent combustion diagram which has been proposed by Peters ${ }^{(1)}$ for turbu- 


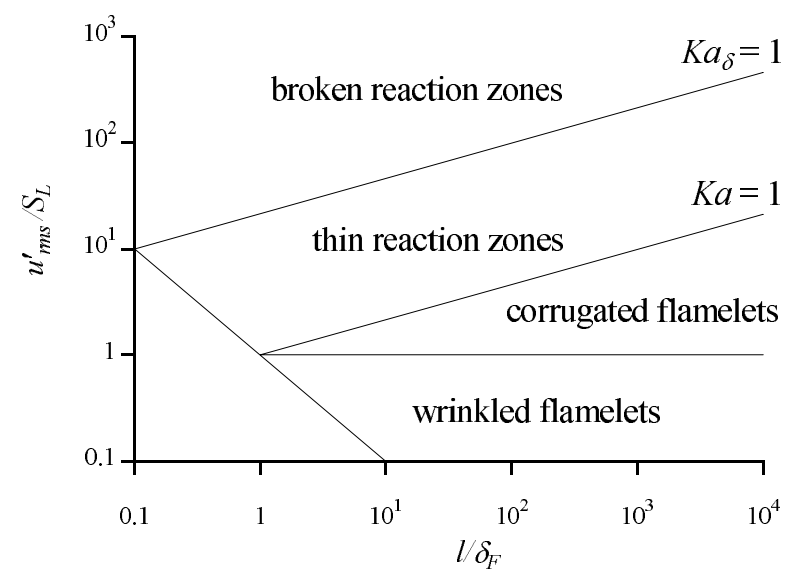

Fig. 1 Turbulent combustion diagram

lent premixed flames. For $u_{r m s}^{\prime} / S_{L}<1$, since flame front is slightly deformed by turbulent motion, this regime is called as wrinkled flamelets. For $u_{r m s}^{\prime} / S_{L}>1$, flame structures might be strongly affected by turbulence. Here, laminar flame structure should be considered before discussing the characteristics of turbulent premixed flames in these regimes. In general, flame thickness is less than $1 \mathrm{~mm}$. The simplest approximation of the flame is to suppose that the flame is infinitely thin sheet compared to length scale of turbulence. However, this approximation becomes incorrect if time scales of turbulence and flames are close to each other.

In combustion chemistry, lots of radicals appear and each radical has an important role in characteristic of the flame. As for simple fuels such as hydrogen, 10 chemical species and 30 elementary reactions are required at least to describe flame correctly. Combustion of hydrocarbons such as methane and propone includes about 50 species and 300 elementary reactions. It has been considered that combustion of gasoline requires several hundreds species and several thousands elementary reactions. These sets of species and elementary reactions are called as detailed kinetic mechanism. A flame is configured by the balance of these elementary reactions which have different chemical time scales. Therefore, internal structure of the flame will be changed when the time scale of turbulence is of the order of that of an elementary reaction. The solid line of $K a=1$ in Fig.1 represents the condition in which time scale of the smallest eddies in turbulence coincides with that of the flame $\left(\delta_{F} / S_{L}\right)$. Note that Kolmogorov eddies of which diameter and azimuthal velocity are Kolmogorov length and velocity are assumed as the smallest eddies in turbulence. For $K a<1$, it is supposed that the internal flame structure does not affected by turbulence and local flame elements are treated as laminar flame with small curvature under the weak strain. The flame structure in this regime is classified to corrugated flamelets. For $K a>1$, a potion of the internal flame structure (preheat zone) is affected by the turbulent motion and each flame element is assumed to be different from laminar flame. Here, this region is called as thin reaction zones in the diagram by Peters ${ }^{(1)}$. However, appellation of this region is depending on the researchers ${ }^{(2),(3)}$.

An ultimate goal of combustion is conversion of chemical bond energy to thermal energy. The width of the conversion region where heat is released is thinner than flame thickness: several tens $\sim$ several hundreds $\mu \mathrm{m}$. For $K a_{\delta}>1$, chemical reaction related to heat release rate will be affected by the turbulent motion. In this regime, it is considered that flame surface is no longer defined. Peters ${ }^{(1)}$ called this regime as broken reaction zones. Since the flame in this regime is scarcely observed even in the real combustors, researchers ${ }^{(2),(3)}$ have a different idea for this regime similar to the thin reaction zones.

As for turbulent diffusion (non-premixed) flame, similar classification has been proposed based on characteristics of turbulence and laminar flame. In the case of turbulent diffusion 
flame, however, classification is difficult compared with turbulent premixed flame because transport of the combustion products should be taken into account. The idea of local flame structure is very important for development of turbulent combustion models both for turbulent premixed and diffusion flames. However, details of the local flame structure is only predicted by theoretical dimensional analysis as described in above because experimental and numerical investigations on the local flame structure have been quite difficult.

\section{DNS of Turbulent Combustion}

To investigate turbulent flame structure and to develop turbulent combustion models, many DNS studies have been conducted extensively. Several reviews concerning to DNS of turbulent combustion are available in literatures ${ }^{(5)-(9)}$. DNS of turbulent combustion can be classified as follows;

I. 2D turbulence and a one-step irreversible reaction,

II. 3D turbulence and a one-step irreversible reaction,

III. 2D turbulence and a detailed kinetic mechanism,

IV. 3D turbulence and a reduced kinetic mechanism,

V. 3D turbulence and a detailed kinetic mechanism.

$2 \mathrm{D}$ or $3 \mathrm{D}$ turbulence simulations with a one-step irreversible reaction in category I and II have been limited in early DNS of turbulent combustion. In recent DNS, a detailed kinetic mechanism, which represents combustion chemistry more correctly, is adopted at least. The most realistic DNS is classified into category V. In these DNS, several tens conservation equations of chemical species should be analyzed with conservation equations of mass, momentum and energy simultaneously. In addition, increment of time advancement should be of the order of nanosecond since very fast elementary reactions are included in the detailed kinetic mechanism. Furthermore, temperature, density and mixture composition change drastically in a quite thin flame and transport/thermal properties also vary with those. From these reasons, DNS of turbulent combustion requires huger computer resources than that of turbulence. Most of recent DNS of turbulent combustion have been conducted in categories III, IV and V for different purposes.

\subsection{Turbulent Premixed Flames}

Fundamental characteristics of turbulent premixed flame have been investigated by DNS of a premixed flame propagating in homogeneous turbulence. First 2D DNS with a realistic kinetic mechanism has been reported for $\mathrm{H}_{2} / \mathrm{O}_{2} / \mathrm{N}_{2}$ turbulent premixed flame by Baum et al. ${ }^{(10)}$ Then, the 2D DNS were extended for hydrogen/air turbulent premixed flames with a detailed kinetic mechanism by Tanahashi et al. ${ }^{(11),(12)}$, for methane/air turbulent premixed flames with a 4-steps reduced kinetic mechanism by Echekki and Chen ${ }^{(13)}$ and for methane/air turbulent premixed flames with a detailed kinetic mechanism including $\mathrm{C} 1$ chemistry by Chen and Echekki ${ }^{(14)}$. Furthermore, DNS of hydrocarbon/air turbulent premixed flames has been conducted for methane/air mixture by Tanahashi et al. ${ }^{(15)}$ and Saito et al. ${ }^{(16),(17)}$, and for propane/air mixture by Haworth et al. ${ }^{(18)}$ with detailed kinetic mechanisms. Although these DNS have been conducted in 2D turbulence, the mechanism of turbulence/flame interaction and characteristics of flames which are curved by turbulent motion have been revealed.

DNS of turbulent combustion with a detailed kinetic mechanism has been extended for 3D cases. The 3D DNS of turbulent combustion have been conducted to investigate vortexflame interaction mechanism in turbulent flame by Tanahashi et al. ${ }^{(19)}$, local flame structure of turbulent flame in thin reaction zones by Tanahashi et al. ${ }^{(20)}$ and three-dimensional flame structure created by three-dimensionality of turbulence by Nada et al. ${ }^{(21)}$. The 3D DNS was also extended for methane-air turbulent premixed flame. Relationship between characteristics of 3D turbulence and flame fronts was investigated by Bell et al. ${ }^{(22)}$ and local extinction of hydrocarbon turbulent flame was discussed by Tanahashi et al. ${ }^{(23)}$ using the 3D DNS. In these DNS of methane-air turbulent premixed flames, reduced kinetic mechanisms, which are 
relatively complex, were taken into account. Furthermore, 3D DNS of heptane-air turbulent premixed flame was reported by Sreedhara and Lakshmisha ${ }^{(24)}$ using a 4-step reduced kinetic mechanism. In addition to these 3D DNS of turbulent premixed flames, DNSs for interaction between vortex pair and premixed flame have been conducted to understand local flame structure in turbulence by simplifying the flow geometry ${ }^{(25)-(28)}$.

\subsection{Turbulent Diffusion Flames}

Although DNS studies on turbulent diffusion flames have been conducted, number of studies is less than that of turbulent premixed flames. Recent progresses in DNS of turbulent diffusion flame have been summarized by Vervish and Poinsot ${ }^{(7)}$. Similar to turbulent premixed flame, a detailed kinetic mechanism is included in DNS of turbulent diffusion flame and DNS of realistic turbulent flames have been conducted in recent years ${ }^{(29)-(32)}$. To investigate elementary process in turbulent diffusion flame, vertex-flame interaction was investigated by Miyauchi et al. ${ }^{(33)}$, and triple flame structure, which is very important for flame stabilization, was clarified by Ruetsch et al. ${ }^{(34)}$ and by Echekki and Chen ${ }^{(35)}$. Auto-ignition mechanism and preferential diffusion effects in turbulent hydrogen/air jet flame were also investigated by DNS $^{(36),(37)}$. Pantano ${ }^{(38)}$ was investigated flame extinction of methane/air jets by DNS. More recently, DNS of laboratory-scale turbulent diffusion flame has been reported by Mizobuchi et al. ${ }^{(39)}$

\subsection{Other Turbulent Combustion Fields}

In addition to the fundamental turbulent combustion field shown in above, various turbulent combustion fields have been investigated by DNS. To investigate fundamental process in engineering applications such as HCCI engines, auto-ignition of a premixed flame in turbulence was investigated by Echekki and $\mathrm{Chen}^{(40)}$ and effects of temperature and composition fluctuations on the auto-ignition were explored by Pires et al. ${ }^{(41)}$, Sankaran et al. ${ }^{(42)}$ and Chen et al. ${ }^{(43),(44)}$. In addition, near-wall behaviors of turbulent flames begun to be studied by $\mathrm{DNS}^{(45),(46)}$ and DNS of radiation/flame interaction in turbulent flames was reported by Wu et al. ${ }^{(47)}$. As described in above, DNS of turbulent combustion tends to be extended for understanding and modeling of many phenomena observed in engineering applications.

\section{LES of Turbulent Flames}

In recent years, numerical analyses of various combustors in engineering applications have been conducted by LES. One of the most important issues in LES of turbulent combustion is treatments of chemical source terms in species conservation equations. In addition to SGS stress models for LES of non-reactive turbulence, SGS combustion models which represent chemical reactions in SGS are required for LES of turbulent combustion. From the treatment of combustion field, LES of turbulent combustion can be classified to three categories: flame tracking method, PDF method and thickened flame method.

In the flame tracking method, dynamics of flame surfaces in turbulence are analyzed by $G$ variable or progress variable. In $G$-equation models, flame surfaces in turbulence are assumed to be infinitely thin. Flame surfaces are represented by constant contour surfaces of $G$ in the $G$-equation models ${ }^{(48)}$. In many cases, turbulent burning velocities in SGS are represented by a monotonically increasing function with respective to the SGS turbulence intensity ${ }^{(49)}$. In several studies such as Im et al. ${ }^{(49)}$, SGS turbulent burning velocities are estimated dynamically by applying a test filter similar to the dynamic procedure in the modeling of SGS stress term. Since $G$ variable changes its value from 0 to 1 discontinuously at the flame front, numerical viscosity should be introduced to stabilize the numerical solution. To improve this problem, a level-set approach is applied for the numerical simulation of the $G$-equation as reported by Wang and Bai ${ }^{(50)}$ and Pitsch and Duchamp De Lageneste ${ }^{(51)}$.

If Lewis number is supposed to be 1 under the one-step reaction, mass fraction of reactant and temperature are related by a certain linear function. In this case, progress variable can 
represent mass fraction of reactant and temperature simultaneously ${ }^{(52),(53)}$. In this method, modeling of the flame surface density is an important issue as is shown by Pierce and Moin ${ }^{(53)}$. To estimate the flame surface density, several procedures used in PDF models for RANS are proposed by Domingo et al. ${ }^{(54)}$ and dynamic procedures have been introduced by Knikker and Veynante ${ }^{(55)}$.

The PDF methods are extensions of turbulent combustion models based on probability density functions (PDF) for RANS modeling. In LES based on PDF models, transport equations of joint probability density functions concerning to mass fractions of chemical species are analyzed instead of conservation equations of those. In these models, joint probability density functions are defined based on mass-weighted average (or Favre average) as described by Jaberi et al. ${ }^{(56)}$ in details. In PDF models, no model for chemical source terms is required, whereas SGS scalar flux models such as those in LES of non-reactive scalar transport and mixing models similar to PDF models for RANS should be developed. The joint probability density functions can be solved by Monte Carlo simulation as shown by Sheikhi et al. ${ }^{(57)}$.

In LES of turbulent combustion, flame thickness is significantly thinner than computational grid size. In the thickened flame model, flame is thickened by variable transformation, which was proposed by Colin et al. ${ }^{(58)}$. In this method, selection of parameters for the variable transformation is very important to obtained stable and correct solutions.

LES described in above have been applied for fundamental combustion fields such as turbulent jet flames to verify the accuracy of turbulent combustion model and numerical procedures, and then extended for realistic combustion field such as gas turbine ${ }^{(59),(60)}$. In addition, modeling of flame structure in thin reaction zone was attempted by Sankaran and Menon ${ }^{(61)}$ and finite reaction rate effects were considered by Kempf et al. ${ }^{(62)}$ to improve application range of LES.

\section{Combined Laser Diagnostics for Turbulent Combustion}

With the development of measurement technology, especially laser diagnostics of combustion fields, experimental results which have high accuracy enough to compare with DNS results described in chapter 3 can be obtained in recent years. In this chapter, recent developments in laser diagnostics of turbulent combustion are summarized.

\subsection{Simultaneous PLIF and PIV}

To investigate turbulent flame structures experimentally, planar laser induced fluorescence (PLIF) of molecules and radicals which are produced by chemical reactions are commonly used ${ }^{(63),(64)}$. PLIF of $\mathrm{CH}$ radicals has been used to investigate characteristics of the flame front in turbulence because $\mathrm{CH}$ radicals are produced at the flame front and have very narrow width enough to represent the reaction zones ${ }^{(65)-(68)}$. Since $\mathrm{OH}$ radicals show high concentration in the burned gas, OH PLIF is useful to distinguish the burned gas from the unburned mixture. Therefore, simultaneous $\mathrm{OH}$ and $\mathrm{CH}$ PLIF has been developed to investigate detailed structure of turbulent flame by Donbar et al. ${ }^{(69)}$, Ratner et al. ${ }^{(70)}$ and Watson et al. ${ }^{(71)}$. To specify the heat release region more precisely, simultaneous $\mathrm{CH}_{2} \mathrm{O}$ and OH PLIF has also reported by Bockle et al. ${ }^{(72)}$ and Ayoola et al. ${ }^{(73)}$.

To understand nature of turbulent flames, measurements of turbulence characteristics in turbulent combustion fields are also important. For this purpose, simultaneous measurements of particle image velocimetry (PIV) and PLIF mentioned above have been developed by many researchers ${ }^{(74)-(78)}$. Most of these researches have been restricted for single radical concentration and two-component velocity measurements on a two-dimensional cross section. This information was not always enough to clarify local flame structure and turbulence characteristics near the flame. In recent years, simultaneous velocity and concentration measurements have been extended to two radical PLIF and two components PIV by Kothnur et al. ${ }^{(79)}$, and to two radical PLIF and three components PIV by Tanahashi et al. ${ }^{(80)}$. Direct comparison between these simultaneous measurements and DNS was also reported recently by Inoue et 
al. ${ }^{(81)}$. In the future, combination of dual-plane PIV ${ }^{(82)-(85)}$ with PLIF is recommended to investigate relations between local flame structure and turbulent motion in details.

\subsection{Time-Resolved PIV and PLIF}

Simultaneous measurements in the above section provides instantaneous information concerning turbulent flames. On the other hand, PIV with quite high temporal resolution has been developed to investigate dynamics of unsteady flows in recent years ${ }^{(86)-(90)}$. This is called as time-resolved or dynamic PIV. The maximum time resolution is $26.7 \mathrm{kHz}$, which has been reposted by Tanahashi et al. ${ }^{(91),(92)}$. This time-resolved PIV also has a possibility to give detailed information of high frequency velocity fluctuation in turbulent flames. As for measurements of dynamical characteristics of turbulent flames, time-resolved OH PLIF measurements by adopting multiple laser system has been developed by Hult et al. ${ }^{(93),(94)}$ and double-pulsed PLIF has been reported for $\mathrm{CH}$ radicals by Lyons et al. ${ }^{(95)}$ and Tanahashi et al. ${ }^{(96)}$, and for $\mathrm{CH}_{2} \mathrm{O}$ by Bladh et al. ${ }^{(97)}$. Combination of these time-resolved PLIFs and timeresolved PIV will clarify characteristics of turbulent flames in which local flame elements interact with turbulence and fluctuate at high frequency in greater details, and gives important information for the development of various turbulent combustion models.

\section{Summary}

In this paper, recent progresses in DNS, LES and laser diagnostics of turbulent combustion are summarized. At present, 3D DNS with detailed kinetic mechanism is limited for hydrogen flames. In the near future, 3D DNS of hydrocarbon fuels such as methane will be possible. However, it should be noted that 3D DNS of turbulent combustion in IC engines for automobiles requires $10^{5} \mathrm{CPU}$ years even by the fastest supercomputer TSUBAME ${ }^{(98)}$ in Japan, which was deployed in April 2006 at Tokyo Institute of Technology and has 100TFlops theoretical peak performance. For the rapid development of high efficiency and low emission combustors, understandings of turbulent combustion and establishment of high accuracy turbulent combustion models for RANS and LES are absolutely imperative.

\section{Acknowledgements}

This work is partially supported by Grant-in-Aid for Scientific Research (A) (No. 152060 23) and (S) (No. 18106004) of Japan Society for the Promotion of Science and by Grant-inAid for Young Scientists (A) (No. 16686011) of the Ministry of Education, Culture, Sports, Science and Technology, Japan.

\section{References}

( 1 ) Peters, N., The Turbulent Burning Velocity for Large-Scale and Small-Scale Turbulence, J. Fluid Mech., Vol. 384 (1999), pp. 107-132.

( 2 ) Libby, P. and Williams, F., Turbulent Reacting Flow, (1980), Academic Press.

( 3 ) Borghi, R., Turbulent Combustion Modeling, Prog. Energy Combust. Sci., Vol. 14 (1988), pp. 245-292.

( 4 ) Poinsot, T. J., Candel S. and Trouve, A., Applications of Direct Numerical Simulation to Premixed Turbulent Combustion, Prog. Energy Combust. Sci., Vol. 21 (1996), pp. 531-576.

( 5 ) Miyauchi, T. and Tanahashi, M., Current State of DNS of Turbulent Combustion, J. Jpn. Soc. Fluid Mech., Vol. 16, No. 3 (1997), pp. 203-208.

( 6 ) Tanahashi, M. and Miyauchi, T., Turbulence/Flame Interaction - DNS of Turbulent Combustion-, J. Jpn. Soc. Fluid Mech., Vol. 16, No. 5 (1997), pp. 391-397.

( 7 ) Vervish, L. and Poinsot, T. J., Direct Numerical Simulation of Non-premixed Turbulent Flames, Annu. Rev. Fluid Mech., Vol. 30 (1998), pp. 655-692.

( 8 ) Numerical Simulation of Combustion, (2000), Maruzen.

( 9 ) Hilbert, R., Tap, F., El-Rabii, H. and Thevenin, D., Impact of Detailed Chemistry and 
Transport Models on Turbulent Combustion Simulations, Prog. Energy Combust. Sci., Vol. 30 (2004), pp. 61-117.

(10) Baum, M., Poinsot, T. J., Haworth, D. C. and Darabiha, N., Direct Numerical Simulation of $\mathrm{H}_{2} / \mathrm{O}_{2} / \mathrm{N}_{2}$ Flames with Complex Chemistry in Two-dimensional Turbulent Flows, $J$. Fluid Mech., Vol. 281 (1994), pp. 1-32.

(11) Tanahashi, M., Miayuchi, T., Nada, Y. and Imamura, Y., Local Flame Structure of a $\mathrm{H}_{2}-$ Air Turbulent Premixed Flame, Trans. Jpn. Soc. Mech. Eng., Vol. 64B, No. 624 (1998), pp. 2662-2668.

(12) Tanahashi, M., Miyauchi, T. and Nada, Y., Turbulence-Flame Interaction in $\mathrm{H}_{2}$-Air Turbulent Premixed Flames, Nensho no Kagaku to Gijitsu, 6 suppl.(1998), pp. 13-22.

(13) Echekki, T. and Chen, J. H., Unsteady Strain Rate and Curvature Effects in Turbulent Premixed Methane-Air Flames, Combust. Flame, Vol. 106 (1996), pp. 184-202.

(14) Chen, J. H., Echekki, T. and Kollmann, W., The Mechanism of Two-Dimensional Pocket Formation in Lean Premixed Methane-Air Flames with Implication to Turbulent Combustion, Combust. Flame, Vol. 116 (1998), pp. 15-48.

(15) Tanahashi, M., Saito, T., Shimamura, M. and Miyauchi, T., Local Extinction and $\mathrm{NO}_{\mathrm{X}}$ Formation in Methane-air Turbulent Premixed Flames, Proc. 2nd Asia-Pacific Conf. on Combust., (1999), pp. 500-503.

(16) Saito, T., Tanahashi, M. and Miyauchi, T., Effects of Turbulence Intensities and Fuel Species in Turbulent Premixed Flames, J. Combust. Soc. Jpn., Vol. 44 (2002), pp. $243-$ 252.

(17) Saito, T., Tanahashi, M. and Miyauchi, T., Local Flame Structure and NO Formation Mechanism in Methane-Air Turbulent Premixed Flames, J. Combust. Soc. Jpn., Vol. 45 (2003), pp. 103-113.

(18) Haworth, D., Cuenot, B., Poinsot, T. and Blint, R., Numerical Simulation of Turbulent Propane-Air Combustion with Non-Homogeneous Reactants, Bulletin of APS, Vol. 43, No. 9 (1998), p.2062.

(19) Tanahashi, M., Fujimura M. and Miyauchi, T., Coherent Fine Scale Eddies in Turbulent Premixed Flames, Proc. Combust. Inst., Vol. 28 (2000), pp. 529-535.

(20) Tanahashi, M., Nada, Y., Ito Y. and Miyauchi, T., Local Flame Structure in the WellStirred Reactor Regime, Proc. Combust. Inst., Vol. 29 (2002), pp. 2041-2049.

(21) Nada, Y., Tanahashi, M. and Miyauchi, T., Effect of Turbulence Characteristics on Local Flame Structure of $\mathrm{H}_{2}$-Air Premixed Flames, J. Turbulence, Vol. 5 (2004), 16.

(22) Bell, J. B., Day, M. S. and Grcar, J. F., Numerical Simulation of Premixed Turbulent Methane Combustion, Proc. Combust. Inst., Vol. 29 (2002), pp. 1987-1993.

(23) Tanahashi, M., Kikuta, S., Nada, Y., Shiwaku, N. and Miyauchi, T., Three-Dimensional DNS of Methane-Air Turbulent Premixed Flames with Realistic Reduced Kinetic Mechanism, Turbulence and Shear Flow Phenomena 4, Vol. 2 (2005), pp. 775-780

(24) Sreedhara, S. and Lakshmisha, K., Direct Numerical Simulation of Autoignition in a Non-Premixed, Turbulent Medium, Proc. Combust. Inst., Vol. 28 (2000), pp. 25-34.

(25) Poinsot, T., Veynante, D. and Candel, S., Quenching Process and Premixed Turbulent Combustion Diagrams, J. Fluid Mech., Vol. 228 (1991), pp. 561-606.

(26) Najm, H. N., and Wyckoff, P. S., Premixed Flame Response to Unsteady Strain Rate and Curvature, Combust. Flame, Vol. 110 (1997), pp. 92-94.

(27) Najm, H. N., Paul, P. H., Mueller, C. J. and Wyckoff, P. S., On the Adequacy of Certain Experimental Observables as Measurements of Flame Burning Rate, Combust. Flame, Vol. 113 (1998), pp. 312-332.

(28) Mantel, T. and Samaniego, J.-H., Fundamental Mechanisms in Premixed Turbulent Flame Propagation via Vortex-Flame Interactions Part II: Numerical Simulation, Combust. Flame, Vol. 118 (1999), pp. 557-582.

(29) Mahalingam, S., Chen, J. H. and Vervish, L., Finite-Rate Chemistry and Transient Effects in Direct Numerical Simulations of Turbulent Non-Premixed Flames, Combust. 
Flame, Vol. 102 (1995), pp. 285-247.

(30) Montgomery, C. J., Kosaly, G. and Riley, J. J., Direct Numerical Solution of Turbulent Nonpremixed Combustion with Multistep Hydrogen/Oxygen Kinetics, Combust. Flame, Vol. 109 (1997), pp. 38-50.

(31) Bedat, B., Egolfopoulos, F. N. and Poinsot, T., Direct Numerical Simulation of Heat Release and $\mathrm{NO}_{\mathrm{X}}$ Formation in Turbulent Non-Premixed Flames, Combust. Flame, Vol. 119 (1999), pp. 69-83.

(32) Li, Y., Tanahashi, M. and Miyauchi, T., Interaction between Hydrogen-Air Diffusion Flame and Large Scale Vortical Structure in Mixing Layer, Proc. 2nd Asia-Pacific Conf. on Combust., (1999), pp. 370-373.

(33) Miyauchi, T., Tanahashi, M., Sasaki, K. and Ozeki, T., Vortex-Flame Interaction in Turbulent Diffusion Flames, Transport Phenomena in Combustion 2 (Ed. S. H. Chan), (1996), p. 1095, Taylor.

(34) Ruetsch, G. R., Vervish, L. and Linan, A., Effect of Heat Release on Triple Flames, Phys. Fluid, Vol. 7 (1995), pp. 1447-1454.

(35) Echekki, T. and Chen, J. H., Structure and Propagation Methanol-Air Triple Flames, Combust. Flame, Vol. 114 (1998), pp. 231-245.

(36) Hilbert, R. and Thevenin, D., Autoignition of Turbulent Non-Premixed Flames Investigated using Direct Numerical Simulations, Combust. Flame, Vol. 128 (2002), pp. 22-37.

(37) Hilbert, R. and Thevenin, D., Influence of Differential Diffusion on Maximum Flame Temperature in Turbulent Nonpremixed Hydrogen/Air Flames, Combust. Flame, Vol. 138 (2004), pp. 175-187.

(38) Pantano, C., Direct Simulation of Non-Premixed Flame Extinction in a Methane Air Jet with Reduced Chemistry, J. Fluid Mech., Vol. 514 (2004), pp. 231-270.

(39) Mizobuchi, Y., Shinjo, J., Ogawa, S. and Takeno, T., A Numerical Study on the Formulation of Diffusion Flame Islands in a Turbulent Hydrogen Jet Lifted Flame, Proc. Combust. Inst., Vol. 30 (2005), pp. 611-619.

(40) Echekki, T. and Chen, J. H., Direct Numerical Simulation of Autoignition in NonHomogeneous Hydrogen-Air Mixtures, Combust. Flame, Vol. 134 (2003), pp. 169-191.

(41) Pires da Cruz, A., Baritaud, T. A. and Poinsot, T. J., Self-Ignition and Combustion Modeling of Initially Nonpremixed Turbulent Systems, Combust. Flame, Vol. 124 (2001), pp. 65-81.

(42) Sankaran, R., Im, H. G., Hawkes, E. R. and Chen, J. H., The Effects of Non-Uniform Temperature Distribution on the Ignition of a Lean Homogeneous Hydrogen-Air Mixture, Proc. Combust. Inst., Vol. 30 (2005), pp. 875-882.

(43) Chen, J. H., Hawkes, E. R., Sankaran, R., Mason, S. D. and Im, H. G., Direct Numerical Simulation of Ignition Front Propagation in a Constant Volume with Temperature Inhomogeneities; I. Fundamental Analysis and Diagnostics, Combust. Flame, Vol. 45 (2006), pp. 128-144.

(44) Chen, J. H., Hawkes, E. R., Sankaran, R., Mason, S. D. and Im, H. G., Direct Numerical Simulation of Ignition Front Propagation in a Constant Volume with Temperature Inhomogeneities; II. Parametric Study, Combust. Flame, Vol. 145 (2006), pp. 145-159.

(45) Jiang, X. and Luo, K. H., Dynamics and Structure of Transitional Buoyant Jet Diffusion Flames with Side-Wall Effects, Combust. Flame, Vol. 133 (2003), pp. 29-45.

(46) Wang, Y. and Trouve, A., Direct Numerical Simulation of Nonpremixed Flame-Wall Interactions, Combust. Flame, Vol. 144 (2006), pp. 461-475.

(47) Wu, Y., Haworth, D. C., Modest, M. F. and Cuenot, B., Direct Numerical Simulation of Turbulence/Radiation Interaction in Premixed Combustion Systems, Proc. Combust. Inst., Vol. 30 (2005), pp. 639-646.

(48) Kerstein, A. R. and Ashurst, W. T., Field Equation for Interface Propagation in an Unsteady Homogeneous Flow Field, Phys. Rev. A, Vol. 37 (1988), pp. 2728-2731.

(49) Im, H. G., Lund, T. S. and Ferziger, J. H., Large Eddy Simulation of Turbulent Front 
Propagation with Dynamic Subgrid Models, Phys. Fluid, Vol. 9 (1997), pp. 3826-3833.

(50) Wang, P. and Bai, X. S., Large Eddy Simulation of Turbulent Premixed Flames using Level-Set G-Equation, Proc. Combust. Inst., Vol. 30 (2005), pp. 582-591.

(51) Pitsch, H. and Duchamp De Lageneste, L., Large-Eddy Simulation of Premixed Turbulent Combustion using a Level-Set Approach, Proc. Combust. Inst., Vol. 29 (2002), pp. 2001-2008.

(52) Boger, M., Veynante, D., Boughanem, H. and Trouve, A., Direct Numerical Simulation Analysis of Flame Surface Density Concept for Large Eddy Simulation of Turbulent Premixed Combustion., Proc. Combust. Inst., Vol. 27 (1998), pp. 917-925.

(53) Pierce, C. D. and Moin, P., Progress-Variable Approach for Large-Eddy Simulation of Non-Premixed Turbulent Combustion, J. Fluid Mech., Vol. 504 (2004), pp. 73-97.

(54) Domingo, P., Vervisch, L., Paye, S. and Hauguel, R., DNS of a Premixed Turbulent V Flame and LES of a Ducted Flame using a FSD-PDF Subgrid Scale Closure with FPI-Tabulated Chemistry, Combust. Flame, Vol. 143 (2005), pp. 266-286.

(55) Knikker, R. and Veynante, D., A Dynamic Flame Surface Density Model for Large Eddy Simulation of Turbulent Premixed Combustion, Phys. Fluid, Vol. 16, No. 11 (2004), pp. L91-L94.

(56) Jaberi, F. A., Colucci, P. J., James, S., Givi, P. and Pope, S. B., Filtered Mass Density Function for Large-Eddy Simulation of Turbulent Reacting Flows, J. Fluid Mech., Vol. 401 (1999), pp. 85-121.

(57) Sheikhi, M. R. H., Drozda, T. G., Givi, P., Jaberi, F. A. and Pope, S. B., Large Eddy Simulation of a Turbulent Nonpremixed Piloted Methane Jet Flame (Sandia Flame D), Proc. Combust. Inst., Vol. 30 (2005), pp. 549-556.

(58) Colin, O., Ducros, F., Veynante, D. and Poinsot, T., A Thickened Flame Model for Large Eddy Simulation of Turbulent Premixed Combustion, Phys. Fluid, Vol. 12, No. 7 (2000), pp. 1843-1863.

(59) Grinstein, F. F. and Fureby, C., LES Studies of the Flow in a Swirl Gas Combustor, Proc. Combust. Inst., Vol. 30 (2005), pp. 1791-1798.

(60) Huang, Y. and Yang, V., Effect of Swirl on Combustion Dynamics in a Lean-Premixed Swirl-Stabilized Combustor, Proc. Combust. Inst., Vol. 30 (2005), pp. 1775-1782.

(61) Sankaran, V. and Menon, S., Subgrid Combustion Modeling of 3-D Premixed Flames in the Thin-Reaction-Zone Regime, Proc. Combust. Inst., Vol. 30 (2005), pp. 575-582.

(62) Kempf, A., Sadiki, A. and Janicka, J., Prediction of Finite Chemistry Effects using Large Eddy Simulation, Proc. Combust. Inst., Vol. 29 (2002), pp. 1979-1985.

(63) Dyer, M. J. and Crosley, D. R., Fluorescence Imaging for Flame Chemistry, Proc. Int. Conf. on Laser '84, (1985), pp. 211-218.

(64) Hanson, R. K., Combustion Diagnostics: Planar Imaging Techniques, Proc. Combust. Inst., Vol. 21 (1986), pp. 1677-1691.

(65) Allen, M., Howe, R. D. and Hanson, R. K., Digital Imaging of Reaction Zones in Hydrocarbon-Air Flames using Planar Laser-Induced Fluorescence of $\mathrm{CH}$ and $\mathrm{C}_{2}, \mathrm{Opt}$ Lett., Vol. 11 (1986), pp. 126-128.

(66) Mansour, M. S., Peters, N. and Chen, Y. C., Investigation of Scalar Mixing in the Thin Reaction Zones Regime using a Simultaneous CH-LIF/Rayleigh Laser Technique, Proc. Combust. Inst., Vol. 27 (1998), pp. 767-773.

(67) Carter, C. D., Donbar, J. M. and Driscoll, J. F., Simultaneous CH Planar Laser-Induced Fluorescence and Particle Imaging Velocimetry in Turbulent Nonpremixed Flames, Appl. Phys. B, Vol. 66 (1998), pp. 129-132.

(68) Paul, P. H. and Najm, H. N., Planar Laser-Induced Fluorescence Imaging of Flame Heat Release Rate, Proc. Combust. Inst., Vol. 27 (1998), pp. 43-50.

(69) Donbar, J. M., Driscoll, J. F. and Carter, C. D., Reaction Zone Structure in Turbulent Nonpremixed Jet Flames-from CH-OH PLIF Images, Combust. Flame, Vol. 122 (2000), pp. 1-19. 
(70) Ratner, A., Driscoll, J. F., Donbar, J. M., Carter, C. D. and Mullin, J. D., Reaction Zone Structure of Non-Premixed Turbulent Flames in the "Intensity Wrinkled" Regime, Proc. Combust. Inst., Vol. 28 (2000), pp. 245-252.

(71) Watson, K. A., Lyons, K. M., Carter, C. D. and Donbar, J. M., Simultaneous Two-Shot CH Planar Laser-Induced Fluorescence and Particle Image Velocimetry Measurements in Lifted CH4/Air Diffusion Flames, Proc. Combust. Inst., Vol. 29 (2002), pp. 19051912.

(72) Bockle, S., Kazenwadel, J., Kunzelmann, T., Shin, D.-I., Schulz, C. and Wolfrum, J., Simultaneous Single-Shot Laser-Based Imaging of Formaldehyde, $\mathrm{OH}$, and Temperature in Turbulent Flames, Proc. Combust. Inst., Vol. 28 (2000), pp. 279-286.

(73) Ayoola, B. O., Balachandra, R., Frank, J. H., Mastorakos, E. and Kaminski, C. F., Spatially Resolved Heat Release Rate Measurements in Turbulent Premixed Flames, Combust. Flame, Vol. 144 (2006), pp. 1-16.

(74) Kalt, P. A. M., Frank, J. H. and Bilger, R. W., Laser Imaging of Conditional Velocities in Premixed Propane-Air Flames by Simultaneous OH PLIF and PIV, Proc. Combust. Inst., Vol. 27 (1998), pp. 751-758.

(75) Rehm, J. E. and Clemens, N. T., The Relationship between Vorticity/Strain and Reaction Zone Structure in Turbulent Non-Premixed Jet Flames, Proc. Combust. Inst., Vol. 27 (1998), pp. 1113-1120.

(76) Han, D. and Mungal, M. G., Simultaneous Measurement of Velocity and CH Layer Distribution in Turbulent Non-Premixed Flames, Proc. Combust. Inst., Vol. 28 (2000), pp. 261-267.

(77) Sinibaldi, J. O., Driscoll, J. F., Mueller, C. J., Donbar, J. M. and Carter, C. D., Propagation Speeds and Stretch Rates Measured along Wrinkled Flames to Assess the Theory of Flame Stretch, Combust. Flame, Vol. 133 (2003), pp. 323-334.

(78) Su, L. K., Sun, O. S. and Mungal, M. G., Experimental Investigation of Stabilization Mechanisms in Turbulent, Lifted Jet Diffusion Flames, Combust. Flame, Vol. 144 (2006), pp. 494-512.

(79) Kothnur, P. S., Tsurikov, M. S., Clemens, N. T., Donbar, J. M. and Carter, C. D., Planar Imaging of $\mathrm{CH}, \mathrm{OH}$, and Velocity in Turbulent Non-Premixed Jet Flames, Proc. Combust. Inst., Vol. 29 (2002), pp. 1921-1927.

(80) Tanahashi, M., Murakami, S., Choi, G.-M., Fukuchi, Y. and Miyauchi, T., Simultaneous CH-OH PLIF and Stereoscopic PIV Measurements of Turbulent Premixed Flames, Proc. Combust. Inst., Vol. 30 (2005), pp. 1665-1672.

(81) Inoue, S., Taka, S., Kato, S., Nada, Y., Tanahashi, M. and Miyauchi, T., Measurement of Local Flame Structures of Turbulent Premixed Flames by Simultaneous CH-OH PLIF and Stereoscopic PIV, Proc. 5th Asia-Pacific Conf. on Combust., (2005), pp. 385-388.

(82) Koahler, C. J. and Kompenhans, J., Fundamentals of Multiple Plane Stereo Particle Image Velocimetry, Exp. Fluids., Vol. 29 (2000), pp. S70-S77.

(83) Hu, H., Saga, T., Kobayashi, T., Taniguchi, N. and Yasuki, M., Dual-Plane Stereoscopic Particle Image Velocimetry: System Set-up and its Application on a Lobed Jet Mixing Flow, Exp. Fluids., Vol. 31 (2001), pp. 277-293.

(84) Mullin, J. A. and Dahm, W. J. A., Dual-Plane Stereo Particle Image Velocimetry (DSPIV) for Measuring Velocity Gradient Fields at Intermediate and Small Scales of Turbulent Flows, Exp. Fluids., Vol. 38 (2005), pp. 185-196.

(85) Ganapathisubramani, B., Longmire, E., Marusic I. and Pothos, S., Dual-Plane PIV Technique to Determine the Complete Velocity Gradient Tensor in a Turbulent Boundary Layer, Exp. Fluids., Vol. 39 (2005), pp. 222-231.

(86) Vogel, A. and Lauterborn, W., Time Resolved Particle Image Velocimetry, Optics and Lasers in Eng., Vol. 9 (1988), pp. 277-294.

(87) Upatnieks, A., Driscoll, J. F. and Ceccio, S. L., Cinema Particle Imaging Velocimetry Time History of the Propagation Velocity of the Base of a Lifted Turbulent Jet Flame, 
Proc. Combust. Inst., Vol. 29 (2002), pp. 1897-1903.

(88) Tanahashi, M., Fukuchi, Y., Fukuzato, K. and Miyauchi, T., Turbulence Measurements by High Resolution Time Series Particle Image Velocimetry. Proc. Thermal Eng. Conf. '02, (2002), pp. 121-122.

(89) Ibsen, C. H., Onofri, F., Solberg, T., Hjertager, B. H. and Tadrist, L., Improved Particle Image Velocimetry Measurements in Gas-Particle Flows with a Dense Wall Layer, Meas. Sci. Technol., Vol. 14 (2003), pp. N9-N12.

(90) Sung, J. and Yoo, J. Y., Near-Wake Vortex Motions behind a Cylinder at Low Reynolds Number, J. Fluids and Structures, Vol. 17 (2003), pp. 261-274.

(91) Tanahashi, M., Fukuchi, Y., Choi, G.-M., Fukuzato, K. and Miyauchi, T., High Spatial Resolution Time-Series PIV for Turbulence Measurement, Proc. 4th Int. Symp. Turbulence, Heat and Mass Transfer, (2003), pp. 245-252.

(92) Tanahashi, M., Fukuchi, Y., Choi, G.-M., Fukuzato, K. and Miyauchi, T., The TimeResolved Stereoscopic Digital Particle Image Velocimetry up to $26.7 \mathrm{kHz}$, Proc. $12 \mathrm{th}$ Int. Symp. Applications of Laser Techniques to Fluid Mechanics, (2004).

(93) Hult, J., Harvey, A. and Kaminski, C. F., Combined High Repetition-Rate OH PLIF and Stereoscopic PIV for Studies of Turbulence-Chemistry Interactions, Optical Society of America, (2003).

(94) Hult, J., Meier, U., Meier, W., Harvey, A. and Kaminski, C. F., Experimental Analysis of Local Flame Extinction in a Turbulent Jet Flame by High Repetition 2-D Laser Techniques and Multi-Scalar Measurements, Proc. Combust. Inst., Vol. 30 (2005), pp. 701-709.

(95) Lyons, K. M., Watson, K. A., Carter, C. D. and Donbar, J. M., On Flame Holes and Local Extinction in Lifted-Jet Diffusion Flames, Combust. Flame, Vol. 142 (2005), pp. 308-313.

(96) Tanahashi, M., Taka, S. and Miyauchi, T., CH Double-Pulsed PLIF Measurement in Turbulent Premixed Flame, 13th Int. Symp. on Appl. Laser Techniques to Fluid Mechanics, (2006).

(97) Bladh, H., Brackmann, C., Dahlander, P., Denbratt, I. and Bengtsson, P. E., Flame Propagation Visualization in a Spark-Ignition Engine using Laser-Induced Fluorescence of Cool-Flame Species, Meas. Sci. Technol., Vol. 16 (2005), pp. 1083-1091.

(98) http://www.gsic.titech.ac.jp/index.html.ja 